\title{
Spontaneous symmetry breaking of gap solitons and phase transitions in double-well traps
}

\author{
M. Trippenbach, ${ }^{1}$ E. Infeld, ${ }^{2}$ J. Gocałek, ${ }^{3}$ M. Matuszewski, ${ }^{4}$ M. Oberthaler, ${ }^{5}$ and B. A. Malomed ${ }^{6}$ \\ ${ }^{1}$ Institute of Theoretical Physics, Physics Department, Warsaw University, Hoża 69, PL-00-681 Warsaw, Poland \\ ${ }^{2}$ Soltan Institute for Nuclear Studies, Hoża 69, PL-00-681 Warsaw, Poland \\ ${ }^{3}$ Institute of Physics, Polish Academy of Sciences, Al. Lotnikw 32/46, Warsaw, Poland \\ ${ }^{4}$ Nonlinear Physics Center and ARC Center of Excellence for Quantum Atom Optics, Research School of Physical Sciences \\ and Engineering, Australian National University, Canberra ACT 0200, Australia \\ ${ }^{5}$ Kirchhoff-Institut für Physik, Im Neuenheimer Feld 227, 69120 Heidelberg, Germany \\ ${ }^{6}$ Department of Physical Electronics, School of Electrical Engineering, \\ Faculty of Engineering, Tel Aviv University, Tel Aviv 69978, Israel \\ (Received 13 February 2008; published 2 July 2008)
}

\begin{abstract}
We study stationary states of a two-dimensional (2D) Bose-Einstein condensate with both attractive and repulsive nonlinearities in a combination of a double-square-well potential in one direction and a perpendicular optical lattice. We look for dual-core solitons in this configuration, focusing on their symmetry-breaking bifurcations. For attractive interactions, without the lattice, a similar analysis was performed [M. Matuszewski et al., Phys. Rev. A 75, 063621 (2007)], where subcritical bifurcation transforming antisymmetric gap solitons into asymmetric ones was found. Here we focus on the effect of an optical lattice and the so created gap solitons. We discover that a phase transition occurs when the lattice depth increases and the additional dimension becomes strongly suppressed. The bifurcation type changes from subcritical (typical for a $2 \mathrm{D}$ system, with hysteresis) to supercritical (typical for a one-dimensional system). An additional advantage of the lattice is that gap solitons exist even for repulsive interactions. In this case we also discover bifurcation of a supercritical type. The analysis is based on a variational approximation, which is surprisingly well verified by numerical results.
\end{abstract}

DOI: 10.1103/PhysRevA.78.013603

PACS number(s): 03.75.Lm, 05.45.Yv, 42.65.Tg

\section{INTRODUCTION}

The Gross-Pitaevskii equation (GPE) provides a powerful model for studying the mean-field dynamics of BoseEinstein condensates [1]. An important example is the prediction of a one-dimensional (1D) gap solitons in a selfrepulsive condensate trapped in a periodic optical lattice [2]. This was realized experimentally in an ultracold gas of ${ }^{87} \mathrm{Rb}$ atoms confined in a cigar-shaped trap [3]. Another example is the prediction of the Josephson effect in a BEC [4]. It was subsequently observed in a condensate trapped in a macroscopic double-well potential [5]. In contrast to hitherto realized Josephson systems in superconductors and superfluids, interactions between tunneling particles play a crucial role in a bosonic junction. The effective nonlinearity induced by the interactions gives rise to new effects in the tunneling. In particular, anharmonic Josephson oscillations were predicted [6-10]. The nonlinearity specific to the BEC gives rise to a self-trapping effect in the form of a self-maintained population imbalance $[5,6]$.

One-dimensional dynamics of a BEC in potentials composed of two rectangular potential wells were studied in several papers [11]. Stationary states with different populations in the two wells are generated by symmetry-breaking bifurcations from symmetric and antisymmetric states, for attractive and repulsive nonlinearity, respectively $[6,10]$. A natural two-dimensional (2D) extension of the double-well configuration is a dual-channel one, with the potential creating two wells in the direction of $x$ extended into parallel troughs along $y[12,13]$. In the case of an attractive nonlinearity, this setting may naturally give rise to dual-core solitons, which are self-trapped in the $y$ direction (similar to the ordinary matter-wave solitons created in a single-core trap [14]). Furthermore, if the ratio of the nonlinearity to the tunneling coupling is large enough the symmetric dual-core soliton may bifurcate into an asymmetric one. This was demonstrated both in the full 2D model [12], and in its 1D counterpart [13].

If the dual-channel potential in 2D geometry is combined with an axial optical lattice, which runs along both potential troughs, it is natural to consider a dual-core gap soliton. In Ref. [13], this was done using the approximation which replaced the corresponding two-dimensional GPE by a pair of linearly coupled $1 \mathrm{D}$ equations. It was demonstrated that a symmetric gap soliton may be stable in this case, and never bifurcates, while asymmetric solitons are generated by a symmetry-breaking bifurcation from antisymmetric ones.

An advantage of the application of an optical lattice is twofold. First, we can consider repulsive interactions, which are easier to realize experimentally. Second, in the case of attractive interactions we can follow the phase transition from two-dimensional geometry, where we predict a subcritical bifurcation, to the one-dimensional case in the strong lattice, where the bifurcation remains supercritical. We predict the parameter regions admitting asymmetric solitons by means of a restricted variational approximation (VA) [16], which allows us to simplify an otherwise very complicated calculation. These results are verified by numerics. We find the agreement to be surprisingly good, for such a restricted variational model.

The paper is organized as follows. The model and the VA are introduced in Sec. II. In Sec. III we analyze the symmetry-breaking bifurcations in both models, with attrac- 


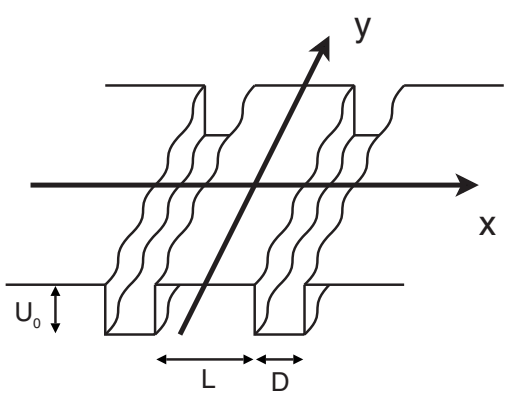

FIG. 1. The shape of the quasi-one-dimensional double-well potential, $U(x, y)$. The wiggles indicate quasi-1D lattice along $y$.

tive and repulsive interactions. In Sec. IV we present the numerical methods and results and Sec. V concludes the paper.

\section{MODEL AND VARIATIONAL APPROXIMATION}

The normalized form of the GPE for the mean-field wave functions $\Psi$ in $2 \mathrm{D}$ geometry is

$$
i \Psi_{t}=-(1 / 2)\left(\Psi_{x x}+\Psi_{y y}\right)+\left[U(x)+\sigma|\Psi|^{2}-\rho \cos (2 y)\right] \Psi,
$$

where $\sigma=+1$ and -1 for the self-repulsive and self-attractive BEC, and $\rho \cos (2 y)$ represents the longitudinal optical-lattice potential. The transverse double-well structure is taken as

$$
U(x)= \begin{cases}0, & |x|<L / 2 \text { and }|x|>L / 2+D, \\ -U_{0}, & L / 2<|x|<L / 2+D,\end{cases}
$$

with $D, U_{0}$, and $L$ being, respectively, the width and depth of each well, and the width of the barrier between them, see Fig. 1 below.

Stationary solutions to Eq. (1) are assumed in the form $\Psi(x, y, t)=e^{-i \mu t} \Phi(x, y)$, where the real function $\Phi(x, y)$ satisfies the equation

$$
\mu \Phi+(1 / 2)\left(\Phi_{x x}+\Phi_{y y}\right)-U(x) \Phi-\sigma \Phi^{3}+\rho \cos (2 y) \Phi=0 .
$$

It can be derived from the Lagrangian,

$$
\begin{aligned}
L_{\text {stat }}= & \iint d x d y\left[\mu \Phi^{2}-(1 / 2)\left(\Phi_{x}^{2}+\Phi_{y}^{2}\right)\right. \\
& \left.-U(x) \Phi^{2}-(\sigma / 2) \Phi^{4}+\rho \cos (2 y) \Phi^{2}\right] .
\end{aligned}
$$

To apply the VA, we follow Ref. [12] and adopt an ansatz consisting of two distinct parts. First, inside each potential trough, i.e., at $|x \mp(L+D) / 2|<D / 2$, the trial function is

$$
\Phi_{ \pm}(x, y)=A_{ \pm} \cos \left(\pi \frac{x \mp(L+D) / 2}{D}\right) \exp \left(-\frac{y^{2}}{2 W^{2}}\right),
$$

where $A_{ \pm}$and $W$ are three variational parameters. This expression implies different amplitudes and a common longitudinal width, $W$, of the wave-function patterns in both troughs. In the $x$ direction, the ansatz (5) emulates the ground-state wave function in an infinitely deep potential box, which vanishes at the edges of the trough, see Fig. 1. In the $y$ direction, the ansatz approximates the self-trapped soliton by a Gaussian profile. Outside the troughs (at $|x|>L / 2$ $+D$ and $|x|<L / 2)$, the ansatz also follows the pattern of quantum mechanics, in the form of a superposition of exponential wave functions:

$$
\Phi(x, y)=\sum_{+,-} A_{ \pm} \exp \left(-\sqrt{-2 \mu}\left|x \mp \frac{L+D}{2}\right|-\frac{y^{2}}{2 W^{2}}\right)
$$

with the same amplitudes $A_{ \pm}$and width $W$ as in Eq. (5). Here $|\cdots|$ stands for modulus. The ansatz is not continuous at the edges of the troughs; however, comparison with numerical findings (see Fig. 4 below) clearly suggest that the VA can be used despite this local discrepancy.

Substitution of expressions (5) and (6) into Eq. (4) upon integration produce the simplified Lagrangian. In this Lagrangian we neglect the contributions from the exponentially decaying functions in the outer region, $|x|>L / 2+D$, we also neglect the term $-(1 / 2) \Phi_{x}^{2}$ in the Lagrangian density. The contribution from the optical-lattice potential is taken into account only inside the troughs. Our approach may seem to be very crude, however, in the past it has so far yielded sensible results $[12,13]$ and the effective Lagrangian so obtained is tractable. In particular, the bifurcation diagram is usually a good approximation. The Lagrangian reads

$$
\begin{aligned}
\frac{2}{D \sqrt{\pi}} L_{\mathrm{eff}}= & \frac{1}{2} \rho W e^{-W^{2}}\left(A_{+}^{2}+A_{-}^{2}\right) \\
& +\sum_{+,-}\left(\frac{\mu+U_{0}}{2} A_{ \pm}^{2} W-\frac{A_{ \pm}^{2}}{8 W}-\frac{3 \sigma}{2^{9 / 2}} A_{ \pm}^{4} W\right) \\
& +\frac{4 \sqrt{-2 \mu}}{D} e^{-\sqrt{-2 \mu}(L+D)} A_{+} A_{-} W .
\end{aligned}
$$

We now define $N_{ \pm} \equiv(3 / 4 \sqrt{2}) A_{ \pm}^{2} W$, and

$$
\lambda \equiv(2 / D) \sqrt{-2 \mu} \exp [-\sqrt{-2 \mu}(L+D)]
$$

$$
N \equiv \frac{N_{+}+N_{-}}{4 \sqrt{\lambda}}, \quad \nu \equiv \frac{N_{+}-N_{-}}{4 \sqrt{\lambda}}, \quad \epsilon \equiv \mu+U_{0} .
$$

The numbers of atoms trapped in the two troughs are proportional to the respective partial norms of the wave function,

$$
\left|\int_{-\infty}^{+\infty} d y \int_{ \pm L / 2}^{ \pm(D+L / 2)} d x[\Phi(x, y)]^{2}\right|=\frac{2 \sqrt{2 \pi}}{3} D N_{ \pm},
$$

hence $\nu$, defined in Eq. (9), measures the population imbalance. In this notation, the Lagrangian (7) simplifies to 


$$
\begin{aligned}
\frac{3}{8 \sqrt{2 \pi \lambda} D} L_{\mathrm{eff}} \equiv & \frac{\epsilon N}{2}-\frac{N}{8 W^{2}}-\sigma \frac{\sqrt{\lambda}}{2} \frac{N^{2}+\nu^{2}}{W} \\
& -s \lambda \sqrt{N^{2}-v^{2}}+\frac{1}{2} \rho N e^{-W^{2}},
\end{aligned}
$$

with $s=+1$ and -1 for the configurations of the antisymmetric and symmetric types (with $A_{+} A_{-}<0$ and $A_{+} A_{-}>0$, respectively).

Our Lagrangian gives rise to variational equations $\partial L / \partial W=\partial L / \partial \nu=\partial L / \partial N=0$,

$$
\begin{gathered}
N+2 \sigma \sqrt{\lambda}\left(N^{2}+\nu^{2}\right) W-4 \rho N W^{4} e^{-W^{2}}=0, \\
\nu\left(-\frac{\sigma}{W}+s \sqrt{\frac{\lambda}{N^{2}-\nu^{2}}}\right)=0, \\
\frac{1}{4 W^{2}}+\sigma \frac{2 \sqrt{\lambda} N}{W}+\frac{2 s \lambda N}{\sqrt{N^{2}-\nu^{2}}}-\rho e^{-W^{2}}=\epsilon .
\end{gathered}
$$

Equation (13) has two solutions: $\nu=0$, which corresponds to symmetric or antisymmetric solitons, and

$$
\nu^{2}=N^{2}-\lambda W^{2},
$$

for asymmetric ones.

For symmetric and antisymmetric solitons, Eqs. (12) and (14), with $\nu=0$, are tantamount to equations that were derived, by means of the VA, for solitons in 1D models with a periodic sinusoidal potential and attractive or repulsive nonlinearity $[19,20]$. In particular, in the latter case (for $\sigma=+1$ ) a known fact is that solutions exist only for $\rho>\rho^{(0)}$ $\equiv e^{2} / 16 \approx 0.462$ (in fact, this constraint predicts, with high accuracy, the edge of the first finite band gap in the linear spectrum induced by the lattice [20]). Results for asymmetric solitons are presented in the next section.

\section{ASYMMETRIC SOLUTIONS}

\section{A. Equations for the bifurcation point}

According to Eq. (13), asymmetric solutions exist in two cases: $\sigma=s=+1$ (repulsion, with the asymmetric branch bifurcating from the antisymmetric one), or $\sigma=s=-1$ (attraction, with the bifurcation from the symmetric branch). Elimination of $\nu^{2}$ in Eqs. (12) and (14) by means of Eq. (15) yields a system of equations for $N$ and $W$,

$$
\begin{gathered}
N+2 \sigma \sqrt{\lambda} W\left(2 N^{2}-\lambda W^{2}\right)=4 \rho N W^{4} e^{-W^{2}}, \\
\frac{1}{4 W^{2}}+\sigma \frac{2 \sqrt{\lambda} N}{W}+\frac{2 s \sqrt{\lambda} N}{W}-\rho e^{-W^{2}}=\epsilon .
\end{gathered}
$$

Taking into account definitions (8) and (9), solutions to Eqs. (16) depend on parameters $L, D, U_{0}$, and $\rho$.

At the bifurcation point, $\nu=0$, Eq. (15) yields $N=\sqrt{\lambda} W$, hence Eqs. (15) generate a system of two equations for two coordinates of the bifurcation point, $\mu$ [via relations (9) and (8)] and $W$,

$$
1+2 \sigma \lambda W^{2}=4 \rho W^{4} e^{-W^{2}}
$$

$$
\frac{1}{4 W^{2}}+2(\sigma+s) \lambda-\rho e^{-W^{2}}=\epsilon .
$$

Without the optical lattice, i.e., for $\rho=0$ (the case considered in Ref. [12]), the first equation in (17) gives the bifurcation point at $N=1 / \sqrt{2}$. To obtain explicit results in the model with $\rho \neq 0$, one can start with an obvious solution to Eqs. (17), at $\lambda=\mu=N=0, \rho=\rho^{(0)}\left(\right.$ recall $\left.\rho^{(0)} \equiv e^{2} / 16\right), U=U_{0}^{(0)} \equiv 1 / 16$, and $W=W^{(0)} \equiv \sqrt{2}$. This solution, which has $N=0$ is, by itself, trivial, but a nontrivial one can be obtained as an expansion around it.

\section{B. Model with self-attraction}

Consider the attraction model corresponding to $\sigma=s=-1$. Then, straightforward analysis of Eqs. (17) for small $\delta \rho=\rho$ $-\rho^{(0)}$ and $\delta U_{0}=U_{0}-U_{0}^{(0)}$ demonstrates that the bifurcation of symmetric solitons (which pertain to $s=-1$, see above) may occur at two values of the norm,

$$
\begin{aligned}
N= & \frac{1}{2 \sqrt{2}} \sqrt{-\left(e^{-2} \delta \rho+\delta U_{0}\right)}\left(2-\frac{1}{2}\left(e^{-2} \delta \rho+\delta U_{0}\right)\right. \\
& \left. \pm \sqrt{15 e^{-2} \delta \rho-\delta U_{0}}\right)
\end{aligned}
$$

the respective value of the width being $W \approx \sqrt{2}\left[1-\left(e^{-2} \delta \rho\right.\right.$ $\left.\left.+\delta U_{0}\right) / 4\right]$. Note that the second term in the large parentheses in Eq. (18) is a small correction to 2, the main correction given by the last term, which demonstrates that theoretically there may be two different bifurcation points. Obviously, expressions (18) are meaningful, i.e., the bifurcation takes place, if

$$
\left(e^{2} / 15\right) \delta U_{0}<\delta \rho<-e^{2} \delta U_{0}
$$

(in other words, $\delta U_{0}$ must be negative, while $\delta \rho$ may have either sign). Numerical calculations imply that only the lower value of $N$ is valid.

A set of bifurcation diagrams in the attraction model, in the form of $\nu(N)$, i.e., curves showing the asymmetry of the dual-core solitons versus the total norm, was generated by a numerical solution of the full system of variational equations (16). The set is displayed in Fig. 2, where a noteworthy feature is the transition from the subcritical shape (backwarddirected one), which is a characteristic of the attraction model without the longitudinal lattice [12] (as well as to the model of dual-core optical fibers [15]), to the simpler supercritical (forward-directed) shape at sufficiently large values of lattice strength $\rho$. Note that the symmetry-breaking bifurcations of dual-core solitons, studied in systems of linearly coupled GPEs including the attractive nonlinearity and lattice potential $[13,17]$, as well as in the system of linearly coupled fiber Bragg gratings [18], are of supercritical type too. The physical significance of the subcritical bifurcation is that it allows bistability of the solitons (the coexistence of stable symmetric and asymmetric ones) in a limited interval of values of $N$.

\section{Model with self-repulsive nonlinearity}

In the case of the self-repulsion, i.e., $\sigma=s=+1$, the expansion of Eqs. (17) predicts the following values of the norm at 


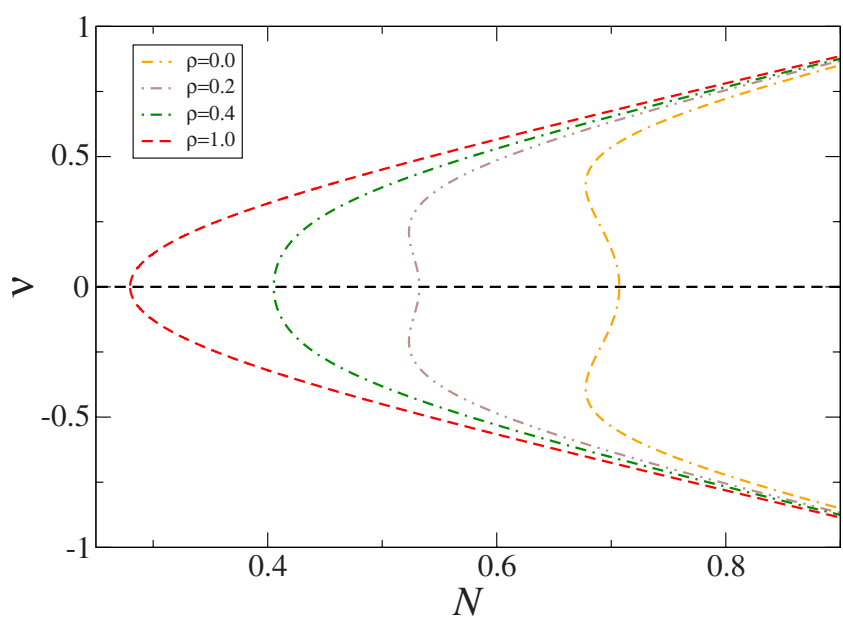

FIG. 2. (Color online) A set of numerically found bifurcation diagrams in the model with attraction, showing degree of a asymmetry of dual-core soliton, $\nu$, as a function of the soliton's total norm, $N$, see Eqs. (9). The diagrams pertain to fixed values of parameters of the transverse double-well configuration, $L=D=1$, $U_{0}=-0.7$ (attractive case), while the strength of the longitudinal optical-lattice potential gradually increases. One can check from the analysis of Eq. (17) that the turning points are at $\nu=0$ and $\pm N / \sqrt{3}$. One can clearly see that the supercritical bifurcation will turn into subcritical bifurcation with increase of the optical lattice strength. It can be interpreted as a phase transition.

which asymmetric gap solitons may bifurcate from the antisymmetric ones (recall antisymmetric solitons corresponds to $s=+1)$ :

$$
\begin{aligned}
N= & \frac{1}{2 \sqrt{2}} \sqrt{e^{-2} \delta \rho+\delta U_{0}}\left(2-\frac{1}{2}\left(e^{-2} \delta \rho+\delta U_{0}\right)\right. \\
& \left. \pm \sqrt{15 e^{-2} \delta \rho-\delta U_{0}}\right)
\end{aligned}
$$

where the notation is the same as in Eq. (18) for the attractive model. This expression predicts the bifurcation in the following region [cf. Eq. (19) in the attraction model]: $-e^{-2} \delta \rho<\delta U_{0}<15 e^{-2} \delta \rho$, which implies $\delta \rho>0$, while $\delta U_{0}$ may be both positive and negative, in contrast with the case of the attraction model, that demanded $\delta U_{0}<0$, while allowing $\delta \rho$ to take either sign. Once again numerical calculations imply that only the lower value of $N$ is valid.

A typical set of bifurcation diagrams in the repulsive model is displayed in Fig. 3, again generated by a numerical solution of the full system of variational equations (16). It is seen that the bifurcation generating asymmetric gap solitons from the antisymmetric ones is always of supercritical type, in compliance with results obtained for the models based on linearly coupled GPEs with the optical-lattice potential and repulsive nonlinearity $[13,17]$. These bifurcation diagrams exist only for $\rho>\rho^{(0)} \equiv e^{2} / 16$, because, as mentioned above, at smaller values of the optical-lattice strength the VA does not predict antisymmetric gap solitons that might give rise to a bifurcation.

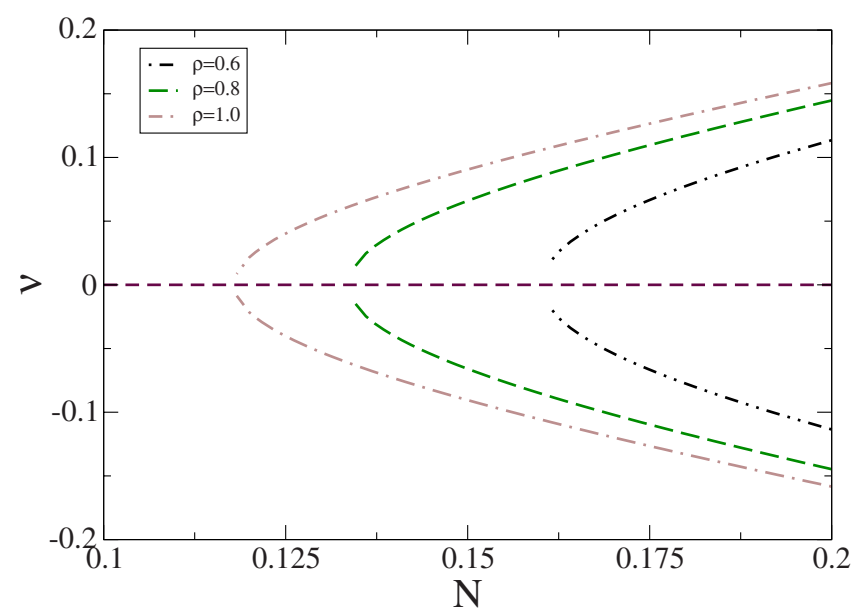

FIG. 3. (Color online) A set of bifurcation diagrams for gap solitons in the model with repulsive nonlinearity, for $L=D=1, U_{0}$ $=4$, and a set of different values of the optical-lattice strength, $\rho$.

\section{NUMERICAL METHODS AND RESULTS}

To verify the above predictions, we solved Eq. (3) numerically, using the imaginary-time relaxation method with a fourth-order Runge-Kutta algorithm. The accuracy of the numerical code was tested by varying computational parameters, namely the mesh density, window size, and time step. These parameters were then fixed at values for which further increase of the accuracy would not lead to a visible change in the final results. This procedure was applied whenever the physical parameter $N$ was changed.

The numerical results are summarized in Figs. 4 and 5 . Comparison of typical asymmetric and symmetric solitons, found from a numerical solution of Eq. (3), with their counterparts predicted by the VA, is presented in Fig. 4. Although at first glance there is discrepancy between the results of these two calculations, the general features are similar. The discrepancies are only observed at the peaks and are a result
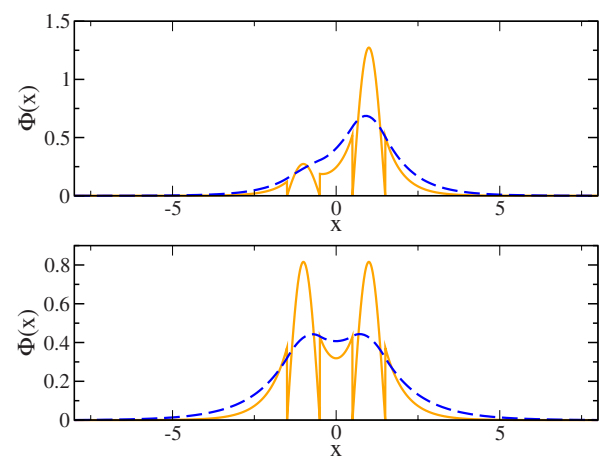

FIG. 4. (Color online) The top and bottom panels demonstrate examples of cross-section profiles, along $y=0$, of stable asymmetric and symmetric gap solitons, as obtained from a numerical solution to Eq. (3) and predicted by the variational approximation (dashed and continuous lines, respectively). Parameters of the double-well potential are $L=D=1, U_{0}=-0.7(\sigma=s=1$, attractive case), and $\rho$ $=1$. Norms of the asymmetric and symmetric solitons are, respectively, $N=0.52$ and $N=0.28$. The asymmetry parameter for the former soliton, see Eqs. (9), is $\nu=0.34$. 


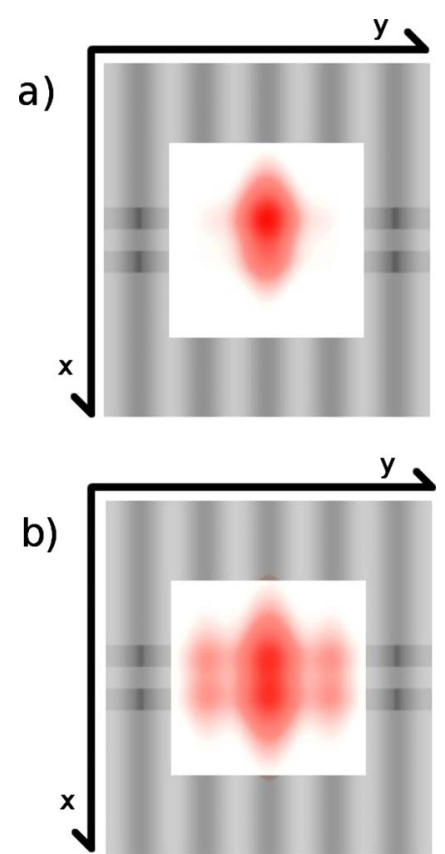

FIG. 5. (Color online) Two-dimensional version of the situation pictured in Fig. 4. Only numerical results following from Eq. (3) are shown. The insets illustrate solitons-the density scale is represented by the intensity of the shading. In the background the horizontal stripes represent double-well structure and vertical modulation visualize optical-lattice potential. Norms of the (a) asymmetric and (b) symmetric solitons are, respectively, $N=0.52$ and $N=0.28$. The asymmetry parameter equal to $\nu=0.34$ is in agreement with the variational predictions of Fig. 2.

of the form of the Ansatz. It is still surprising that the agreement is so good in spite of the simplicity of the model. A similar restricted Ansatz was used in Ref. [12] and discrepancies of the wave functions were of the same order. Nevertheless we were able to predict the form of the bifurcation diagram correctly. Here also the variational calculation helped us to predict the values of the parameters and where to expect symmetric and asymmetric solutions. Even the asymmetry parameter is in agreement with the variational predictions of Fig. 2.

To better visualize the situation we also present a twodimensional density plot (Fig. 5) obtained numerically.

\section{CONCLUSIONS}

We have introduced a 2D model for self-attractive and self-repulsive BECs, which combines a double-well potential in the transverse direction, and a periodic potential along the longitudinal direction. The analysis involved symmetrybreaking bifurcations for dual-core solitons. Our results were obtained by means of a restricted variational approximation. We so created bifurcation diagrams and predicted the values of parameters that should yield symmetric, antisymmetric, and asymmetric solitons. Agreement with numerical solutions was good. However, the exact shape of the solitons was only reproduced outside the troughs. In the case of a repulsive nonlinearity, the bifurcation is of supercritical type, while in the model with attraction an increase of the opticallattice strength leads to a phase transition from subcritical bifurcation to a supercritical one.

\section{ACKNOWLEDGMENTS}

M.T. acknowledges the support of the Polish Government Research Grant for 2006-2009. E.I. and M.M. acknowledge the support of the Polish Government Research Grant for 2007-2010 and 2007-2009. The work of B.A.M. was partially supported by the Israel Science Foundation through Excellence-Center Grant No. 8006/03. B.A.M. would like to thank Soltan Institute for Nuclear Studies, Warsaw, for an invitation in 2007. B.A.M. and M.O. acknowledge the support by German-Israel Foundation through Grant No. 149/ 2006.
[1] F. Dalfovo, S. Giorgini, L. P. Pitaevskii, and S. Stringari, Rev. Mod. Phys. 71, 463 (1999).

[2] F. Kh. Abdullaev, B. B. Baizakov, S. A. Darmanyan, V. V. Konotop, and M. Salerno, Phys. Rev. A 64, 043606 (2001); I. Carusotto, D. Embriaco, and G. C. La Rocca, ibid. 65, 053611 (2002); B. B. Baizakov, V. V. Konotop, and M. Salerno, J. Phys. B 35, 5105 (2002); E. A. Ostrovskaya and Y. S. Kivshar, Phys. Rev. Lett. 90, 160407 (2003).

[3] B. Eiermann, Th. Anker, M. Albiez, M. Taglieber, P. Treutlein, K.-P. Marzlin, and M. K. Oberthaler, Phys. Rev. Lett. 92, 230401 (2004).

[4] J. Javanainen, Phys. Rev. Lett. 57, 3164 (1986); A. Smerzi, S. Fantoni, S. Giovanazzi, and S. R. Shenoy, ibid. 79, 4950 (1997); S. Giovanazzi, A. Smerzi, and S. Fantoni, ibid. 84, 4521 (2000); E. A. Ostrovskaya, Yuri S. Kivshar, Mietek Lisak, Bjorn Hall, Federica Cattani, and Dan Anderson, Phys. Rev. A 61, 031601(R) (2000); K. W. Mahmud, J. N. Kutz, and W. P. Reinhardt, ibid. 66, 063607 (2002).
[5] M. Albiez, R. Gati, J. Folling, S. Hunsmann, M. Cristiani, and M. K. Oberthaler, Phys. Rev. Lett. 95, 010402 (2005).

[6] A. Smerzi, S. Fantoni, S. Giovanazzi, and S. R. Shenoy, Phys. Rev. Lett. 79, 4950 (1997); S. Raghavan, A. Smerzi, S. Fantoni, and S. R. Shenoy, Phys. Rev. A 59, 620 (1999).

[7] J. Javanainen, Phys. Rev. Lett. 57, 3164 (1986).

[8] M. W. Jack, M. J. Collett, and D. F. Walls, Phys. Rev. A 54, R4625 (1996).

[9] I. Zapata, F. Sols, and A. J. Leggett, Phys. Rev. A 57, R28 (1998).

[10] G. J. Milburn, J. Corney, E. M. Wright, and D. F. Walls, Phys. Rev. A 55, 4318 (1997).

[11] P. Zin, E. Infeld, M. Matuszewski, G. Rowlands, and M. Trippenbach, Phys. Rev. A 73, 022105 (2006); E. Infeld, P. Zin, J. Gocalek, and M. Trippenbach, Phys. Rev. E 74, 026610 (2006); for a review, see R. Gati and M. Oberthaler, J. Phys. B 40, R61 (2007).

[12] M. Matuszewski, B. A. Malomed, and M. Trippenbach, Phys. 
Rev. A 75, 063621 (2007).

[13] A. Gubeskys and B. A. Malomed, Phys. Rev. A 75, 063602 (2007).

[14] K. E. Strecker et al., Nature (London) 417, 150 (2002); L. Khaykovich et al., Science 296, 1290 (2002).

[15] E. M. Wright, G. I. Stegeman, and S. Wabnitz, Phys. Rev. A 40, 4455 (1989); N. Akhmediev and A. Ankiewicz, Phys. Rev. Lett. 70, 2395 (1993); P. L. Chu, B. A. Malomed, and G. D. Peng, J. Opt. Soc. Am. B 10, 1379 (1993).

[16] V. M. Pérez-García, Humberto Michinel, J. I. Cirac, M. Lewenstein, and P. Zoller, Phys. Rev. A 56, 1424 (1997); B. A. Malomed, in Progress in Optics, edited by E. Wolf (North-
Holland, Amsterdam, 2002), Vol. 43, p. 71.

[17] A. Gubeskys and B. A. Malomed, Phys. Rev. A 76, 043623 (2007).

[18] W. Mak, B. A. Malomed, and P. L. Chu, J. Opt. Soc. Am. B 15, 1685 (1998); Y. J. Tsofe and B. A. Malomed, Phys. Rev. E 75, 056603 (2007).

[19] B. A. Malomed, Z. H. Wang, P. L. Chu, and G. D. Peng, J. Opt. Soc. Am. B 16, 1197 (1999).

[20] S. K. Adhikari and B. A. Malomed, Europhys. Lett. 79, 50003 (2007); S. K. Adhikari and B. A. Malomed, Phys. Rev. A 76, 043626 (2007). 